\title{
On identifying codes in the king grid that are robust against edge deletions
}

\author{
Iiro Honkala* and Tero Laihonen ${ }^{\dagger}$ \\ Department of Mathematics, \\ University of Turku, \\ 20014 Turku, Finland \\ e-mail: \{honkala,terolai\}@utu.fi
}

Submitted: Aug 20, 2007; Accepted: Dec 14, 2007; Published: Jan 1, 2008

Mathematics Subject Classification: 05C70,05C90,94C12,94B65

\begin{abstract}
Assume that $G=(V, E)$ is an undirected graph, and $C \subseteq V$. For every $v \in V$, we denote $I_{r}(G ; v)=\{u \in C: d(u, v) \leq r\}$, where $d(u, v)$ denotes the number of edges on any shortest path from $u$ to $v$. If all the sets $I_{r}(G ; v)$ for $v \in V$ are pairwise different, and none of them is the empty set, the code $C$ is called $r$-identifying. If $C$ is $r$-identifying in all graphs $G^{\prime}$ that can be obtained from $G$ by deleting at most $t$ edges, we say that $C$ is robust against $t$ known edge deletions. Codes that are robust against $t$ unknown edge deletions form a related class. We study these two classes of codes in the king grid with the vertex set $\mathbb{Z}^{2}$ where two different vertices are adjacent if their Euclidean distance is at most $\sqrt{2}$.
\end{abstract}

Keywords: Identifying code, edge deletion, king grid, optimal code.

\section{Introduction}

Assume that $G=(V, E)$ is a simple, connected undirected graph with vertex set $V$ and edge set $E$.

The distance between two vertices $u$ and $v$ of $G$ is defined to be the number of edges on any shortest path from $u$ to $v$, and is denoted by $d(u, v)$ (or by $d_{G}(u, v)$ if we wish to emphasize which graph we are referring to). We denote

$$
B_{r}(v)=B_{r}(G ; v)=\{u \in V \mid d(u, v) \leq r\} .
$$

\footnotetext{
${ }^{*}$ Research supported by the Academy of Finland under grant 210280.
}

${ }^{\dagger}$ Research supported by the Academy of Finland under grant 111940. 
A nonempty subset of vertices, $C \subseteq V$, is called a code and its elements are codewords.

If $C$ is a code and $v \in V$, we denote

$$
I_{r}(v)=I_{r}(G ; v)=C \cap B_{r}(v) .
$$

A code is called $r$-identifying (in $G$ ) if the sets $I_{r}(v)$ are nonempty and pairwise different for all $v \in V$. In a closely related problem of $r$-locating-dominating sets, the sets $I_{r}(v)$ must also be nonempty and different, but now only for $v \notin C$. The two problems have been widely studied, see $[1,2,3,5,6,7,8,9,10,24,25,28,29,30]$ and for more the web-site [23]. Among the most studied underlying graphs are the square grid, the triangular grid, the king grid, trees, cycles and hypercubes. The original motivation for identifying codes is locating faulty processors in a multiprocessor system [18]. They can also be applied to sensor networks [26]. In both applications, the goal is to find as small an identifying code as possible.

It is natural (see, e.g., [27]) to study codes that remain identifying, for instance, when some edges are deleted in the underlying graph. In this paper we consider the following two classes of robust identifying codes from [16]. For other types of robust identifying codes, see also [14], [11], [12], [13], [17], [19], [20], [21], [22], [26].

Definition 1 An r-identifying code $C \subseteq V$ is called robust against $t$ known edge deletions, if $C$ is $r$-identifying in every graph $G^{\prime}$ that can be obtained from $G$ by deleting at most $t$ edges.

Definition 2 An r-identifying code $C \subseteq V$ in $G$ is called robust against $t$ unknown edge deletions, if it has the following property:

if $u$ and $v$ are any two different vertices of $V$ and $G_{1}$ and $G_{2}$ are any two (possibly the same) graphs each obtained from $G$ by deleting at most $t$ edges, then $I_{r}\left(G_{1} ; u\right) \neq I_{r}\left(G_{2} ; v\right)$ and $I_{r}\left(G_{1} ; u\right) \neq \emptyset$.

Here the idea is that we know that at most $t$ edges have been deleted from $G$, but do not know which ones, but although we do not know what the resulting graph $G^{\prime}$ is, $I_{r}\left(G^{\prime} ; u\right)$ gives enough information to uniquely determine $u$.

In this paper we study the king grid $K$, whose vertex set is $\mathbb{Z}^{2}$ and in which two different vertices are adjacent if their Euclidean distance is at most $\sqrt{2}$. The king grid is a mathematically attractive model, because a ball of radius $r$ has a particularly simple form, being a $(2 r+1) \times(2 r+1)$ square of vertices.

Denote by $Q_{n}$ the set of vertices $(x, y) \in V$ with $|x| \leq n$ and $|y| \leq n$. Then the density of a code $C$ is defined as

$$
D(C)=\limsup _{n \rightarrow \infty} \frac{\left|C \cap Q_{n}\right|}{\left|Q_{n}\right|} \text {. }
$$

We try to determine how small the densities of codes that are robust against known or unknown edge deletions can be.

From now on, we always assume that $t \geq 1$ and $r \geq 1$. 


\section{Unknown edge deletions}

We begin by looking at identifying codes that are robust against unknown edge deletions for different values of radius and number of deletions.

\subsection{Codes with $t=1$}

The case $t=r=1$ has been discussed in [16], so we focus on $r>1$.

The following result from [15] turns out to be useful in what follows when we bound from below the density of a code.

Theorem 3 Assume that $C \subseteq \mathbb{Z}^{2}$ is a code in the king grid. Let $S=\left\{s_{1}, s_{2}, \ldots, s_{k}\right\}$ be a subset containing $k$ different vertices. For each $i=1,2, \ldots, k$ we choose a real number $w_{i}$, which we call the weight of $s_{i}$ and denote it by $w\left(s_{i}\right)$. For all subsets $A$ of $S$ we denote

$$
w(A)=\sum_{a \in A} w(a)
$$

If for all $v \in \mathbb{Z}^{2}$ we have

$$
w((v+C) \cap S) \geq m,
$$

and $w_{1}+w_{2}+\ldots+w_{k}>0$, then the density $D$ of $C$ satisfies

$$
D \geq \frac{m}{w_{1}+w_{2}+\ldots+w_{k}} .
$$

In the sequel we also need the following observation of the king grid.

Lemma 4 Let $(i, j) \in \mathbb{Z}^{2}$ be arbitrary and $r \geq 2$. Then $\left.i\right)$ there are three edge-disjoint paths of length $r$ from $(i, j)$ to each of the points $(i-r+2, j+r),(i-r+3, j+r)$, $\ldots,(i+r-2, j+r)$, and ii) there are two edge-disjoint paths of length $r$ to the points $(i-r+1, j+r)$ and $(i+r-1, j+r)$. Moreover, iii) there exists three edge-disjoint paths of length at most $r$ from $(i, j)$ to the points $(i-r+1, j+r-1),(i-r+2, j+r-1), \ldots$, $(i+r-1, j+r-1)$.

Proof. Consider first i). The path needs to go further up in each step, and the only possible moves that can be used on such a path are $L$, a move to the left and up, $U$ a move up, and $R$, a move to the right and up. The sequence $R^{k+1} U^{r-k-2} L$ (i.e., we first use the move $R k+1$ times, then $U r-k-2$ times and finally $L$ once) takes us from $(i, j)$ to $(i+k, j+r)$ for all $k$ with $0 \leq k \leq r-2$, and so do the sequences $U R^{k} U^{r-k-1}$ and $L U^{r-k-2} R^{k+1}$. It is easy to check that these three paths are edge-disjoint. The other cases in i) are symmetric. To see ii) for the point $(i+r-1, j+r)$, we simply use the sequences $R^{r-1} U$ and $U R^{r-1}$. The other case is again symmetric. Let us now look at the case iii). The claim is trivial for $r=2$, so assume $r \geq 3$. By i) (replace $r$ by $r-1$ ), it suffices to consider the symmetric points $(i-r+1, j+r-1)$ and $(i+r-1, j+r-1)$ 
and the symmetric points $(i-r+2, j+r-1)$ and $(i+r-2, j+r-1)$. The point $(i+r-1, j+r-1)$ has the following three edge-disjoint paths of length at most $r ; R^{r-1}$, $U R^{r-2} S$, where $S$ is denotes the step to the right, and the reflection of the latter one with respect to $R^{r-1}$. The point $(i+r-2, j+r-1)$ already has, by ii) (replace $r$ by $r-1$ again), two edge-disjoint paths to $p$. The third one is $L R^{r-2} S$.

The following theorem gives the smallest possible density for the case $t=1$ and $r>1$.

Theorem 5 Assume that $r>1$. In the king grid the smallest possible density of an $r$-identifying code that is robust against one unknown edge deletion is $\frac{1}{4 r}$.

Proof. We know from [4] that the density of an $r$-identifying code in $K$ must be at least $\frac{1}{4 r}$ for all $r>1$. In this case, there is also a simple proof that follows from the previous theorem: see the proof of Theorem 10.

It therefore suffices to prove that the code

$$
C=\{(x, y) \mid y-x \equiv 0 \quad(\bmod 2 r), x \equiv 0 \quad(\bmod 2)\}
$$

is an $r$-identifying code that is robust against one unknown edge deletion. Notice that we can change the condition $x \equiv 0 \quad(\bmod 2)$ to $y \equiv 0 \quad(\bmod 2)$ without changing the code C.

Assume that $p=\left(x_{p}, y_{p}\right)$ and $q=\left(x_{q}, y_{q}\right)$ are two different vertices. We show that $I_{r}\left(K^{\prime} ; p\right) \neq I_{r}\left(K^{\prime \prime} ; q\right)$ whenever $K^{\prime}$ and $K^{\prime \prime}$ are two (not necessarily different) graphs each obtained by deleting at most one edge from $K$.

The code is clearly symmetric with respect to the line $y=-x$; so we can without loss of generality assume that $y_{p}>y_{q}$.

Assume first that $y_{p}=y_{q}+1$. Either $y_{p}+r$ or $y_{q}-r$ is even: without loss of generality, $y_{p}+r$ is even. Then every $(2 r)$-th point on the line $y=y_{p}+r$ is a codeword. Hence i) one of the $2 r-1$ points $p+(-r+1, r), p+(-r+2, r), \ldots, p+(r-1, r)$ is in $C$ or ii) both $p+(-r, r)$ and $p+(r, r)$ are in $C$.

If i) holds, then that codeword belongs to $I_{r}\left(K^{\prime} ; p\right)$, because (by the previous lemma) there are at least two edge-disjoint paths to this codeword from $p$ (and at most one edge is deleted in order to obtain $K^{\prime}$ from $K$ ). Clearly, this codeword does not belong to $I_{r}(K ; q)$ and therefore not to $I_{r}\left(K^{\prime \prime} ; q\right)$ either.

If ii) holds, then there are edge-disjoint paths from $p$ to $p+(-r, r)$ and $p+(r, r)$, and therefore at least one of the points $p+(-r, r)$ and $p+(r, r)$ belongs to $I_{r}\left(K^{\prime} ; p\right)$. Again, this codeword does not belong to $I_{r}\left(K^{\prime \prime} ; q\right)$, and we are done.

Assume therefore that $y_{p} \geq y_{q}+2$. Then either the line $y=y_{p}+r$ or $y=y_{p}+r-1$ contains codewords, and a similar argument works (use the part iii of Lemma 4 in case of the line $y=y_{p}+r-1$ ).

Finally, we need to check that for all $p=\left(x_{p}, y_{p}\right) \in \mathbb{Z}^{2}$ we have $I_{r}\left(K^{\prime} ; p\right) \neq \emptyset$ in all graphs $K^{\prime}$ obtained from $K$ by deleting at most one edge. Again, either the line $y=y_{p}+r$ or $y=y_{p}+r-1$ contains codewords, and the same argument as above proves the claim. 


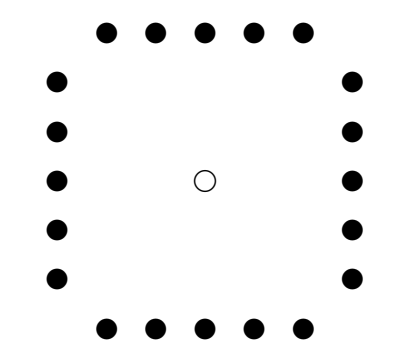

Figure 1: If the open circle is not in $C$, then all the solid circles are.

\subsection{Codes with $t=2$ and $r=1$}

Assume that $C$ is a 1-identifying code in the infinite king grid and that it is robust against two unknown edge deletions. Obviously $\left|B_{1}((i, j)) \triangle B_{1}((i+1, j))\right|=6$, and by definition, all three elements in $B_{1}((i, j)) \backslash B_{1}((i+1, j))$ or all three elements in $B_{1}((i+1, j)) \backslash B_{1}((i, j))$ are in $C$. Because this holds for all pairs $(i, j)$, we conclude that if $u \notin C$, then all the points $u+(-3,2), u+(-3,1), u+(-3,0), u+(-3,-1)$ and $u+(-3,-2)$ are in $C$, and so are the points $u+(3,2), u+(3,1), u+(3,0), u+(3,-1), u+(3,-2)$. We can also reverse the roles of the $x$ - and $y$-coordinates in the argument, and see that if the open circle in Figure 1 is not in $C$, then all the 20 solid circles must be in the code.

We denote

$$
F(i, j)=\{(i, j),(i, j+3),(i+3, j+3),(i+3, j)\},
$$

and call it the frame at $(i, j)$. Each frame contains at least two codewords, and if there are only two codewords, then the codewords are diagonally opposite. Denote

$$
\mathcal{F}_{k}=\{F(i, j)|| F(i, j) \cap C \mid=k\}
$$

for $k=2,3,4$. We would like to show that in average, each frame contains at least three codewords. For that purpose, we devise a counting scheme which shows how the frames with only two codewords can be averaged out.

We ask the frames in $\mathcal{F}_{4}$ to give votes to the frames in $\mathcal{F}_{2}$ according to the following rule:

Assume that $F(i, j) \in \mathcal{F}_{4}$. If the list

$$
\begin{gathered}
F(i+1, j), F(i+2, j), F(i, j+1), F(i, j-1), F(i+1, j+1), \\
F(i+1, j-1), F(i+2, j+1), F(i+2, j-1)
\end{gathered}
$$

contains a frame that belongs to $\mathcal{F}_{2}$, then $F(i, j)$ gives one vote to the first frame in $\mathcal{F}_{2}$ on the list. Otherwise, we take the first of the two sets $\{F(i, j+$ $2), F(i, j-2)\}$ and $\{F(i+1, j+2), F(i+1, j-2)\}$ that contains at least one element of $\mathcal{F}_{2}$, and $F(i, j)$ gives half a vote to each element of $\mathcal{F}_{2}$ in that set. If neither of the two sets contains an element of $\mathcal{F}_{2}$, then no votes are given. 
Clearly, each frame $F(i, j) \in \mathcal{F}_{4}$ gives at most one vote in all.

Each frame has been labelled by its lower-left corner. Using this labelling we can illustrate the voting rule above by the figure

$\begin{array}{lll}6 & 7 & \\ 3 & 4 & 5 \\ 0 & 1 & 2 \\ 3 & 4 & 5 \\ 6 & 7 & \end{array}$

where 0 is the frame itself. Using Figure 1 one immediately checks that the two frames labelled by 3 (resp. 4, 5) cannot both simultaneously belong to $\mathcal{F}_{2}$. During the voting the frame 0 first looks at the numbers $1,2,3,4$ and 5 , and if there is a frame from $\mathcal{F}_{2}$, the one with the smallest label in this array gets one vote. If none of these eight frames belongs to $\mathcal{F}_{2}$, then we look at the two 6 's. If at least one of them belongs to $\mathcal{F}_{2}$, then each of them that belongs to $\mathcal{F}_{2}$ gets half a vote. Otherwise, we look at the two 7's and do the same thing. In particular, we see that the voting rule is symmetric with respect to the middle horizontal line in the array.

Lemma 6 Each frame in $\mathcal{F}_{2}$ gets at least one vote in all.

Proof. Without loss of generality, we can assume that $F(i, j) \in \mathcal{F}_{2}$, and that the two non-codewords in $F(i, j)$ are in the lower-left and upper-right corners (in the other case, we just go up instead of going down in what follows: as explained above, the voting rule is symmetric in this sense). Then using Figure 1 we have Constellation 1 in Figure 2 where the open circles denote the two non-codewords in $F(i, j)$ and solid circles denote codewords. The point $(i, j)$ is the non-codeword in the middle.

If $F(i-1, j)$ is in $\mathcal{F}_{4}$, i.e., $(i-1, j)$ is in $C$, then $F(i-1, j)$ gives $F(i, j)$ one vote, and we are done. Assume therefore that $(i-1, j)$ is not in $C$. Then by Figure $1,(i+2, j-2)$, $(i+2, j-1),(i+2, j+1)$ and $(i+2, j+2)$ are all in $C$.

If $F(i-2, j)$ is in $\mathcal{F}_{4}$, i.e., $(i-2, j)$ is in $C$, then (because $\left.F(i-1, j) \notin \mathcal{F}_{2}\right) F(i-2, j)$ gives $F(i, j)$ one vote, and we are done. Assume therefore that $(i-2, j)$ is not in $C$. Then all the points $(i+1, j-2),(i+1, j-1),(i+1, j+1)$ and $(i+1, j+2)$ are in $C$, and we have Constellation 2 in Figure 2.

If $F(i, j-1) \in \mathcal{F}_{4}$, i.e., $(i, j-1)$ is in $C$, then it $F(i, j-1)$ gives $F(i, j)$ one vote, because it does not give any votes to $F(i+1, j-1)$ or $F(i+2, j-1)$. Assume therefore that $(i, j-1)$ is not in $C$. Then $(i-2, j+2),(i-1, j+2),(i-2, j-4),(i-1, j-4)$, $(i, j-4),(i+1, j-4),(i+2, j-4)$ and $(i+3, j-3)$ are all in $C$.

If $(i-1, j-1)$ is in $C$, then $F(i-1, j-1)$ gives $F(i, j)$ one vote; so assume that $(i-1, j-1)$ is not in $C$.

If $(i-2, j-1)$ is in $C$, then $F(i-2, j-1)$ gives $F(i, j)$ one vote; so assume that $(i-2, j-1)$ is not in $C$. Now we have Constellation 3 in Figure 3. From the definition, it immediately follows that $\left|I_{1}((i-1, j))\right| \geq 3$ and $\left|I_{1}((i-1, j-1))\right| \geq 3$, and therefore 


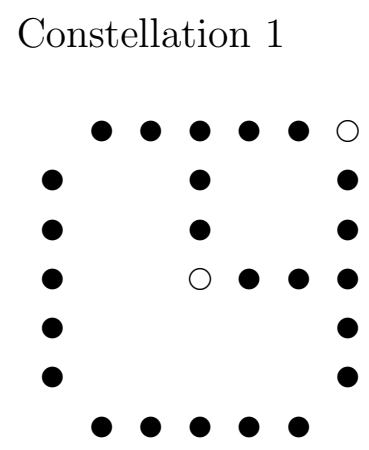

Constellation 2

Figure 2: Two constellations.

the five points $(i-2, j-2),(i-1, j-2),(i, j-2),(i-2, j+1),(i-1, j+1)$ are all in $C$. Now both $F(i, j-2)$ and $F(i-1, j-2)$ gives $F(i, j)$ half a vote, and we are done.

Theorem 7 The smallest possible density of a 1-identifying code in the king grid that is robust against two unknown edge deletions is $3 / 4$.

Proof. It is easy to check that the code in Constellation 4 in Figure 3 is a 1-identifying code that is robust against two unknown edge deletions and has density $3 / 4$.

Assume that $C$ is any 1-identifying code that is robust against two unknown edge deletions. Recall that $Q_{n}=\left\{(x, y) \in \mathbb{Z}^{2}|| x|\leq n| y \mid, \leq n\right\}$. First, every point in $Q_{n}$ is contained in exactly four frames $F(i, j)$. Second, by the previous lemma,

$$
\begin{aligned}
& \# \text { of frames } F(i, j) \in \mathcal{F}_{2} \text { with }(i, j) \in Q_{n-3} \\
& \leq \text { \# of votes received by } F(i, j) \text { with }(i, j) \in Q_{n-3} \\
& \leq \text { \# of votes given by } F(i, j) \text { with }(i, j) \in Q_{n-1} \\
& \leq \text { \# of frames } F(i, j) \in \mathcal{F}_{4} \text { with }(i, j) \in Q_{n-1} .
\end{aligned}
$$

Using these two observations we get

$$
\begin{aligned}
4\left|C \cap Q_{n}\right| \geq & \sum_{(i, j) \in Q_{n-3}}|F(i, j) \cap C| \\
= & 3\left|Q_{n-3}\right|+\left|\left\{(i, j) \in Q_{n-3} \mid F(i, j) \in \mathcal{F}_{4}\right\}\right| \\
& -\left|\left\{(i, j) \in Q_{n-3} \mid F(i, j) \in \mathcal{F}_{2}\right\}\right| \\
\geq & 3\left|Q_{n-3}\right|+\left|\left\{(i, j) \in Q_{n-3} \mid F(i, j) \in \mathcal{F}_{4}\right\}\right| \\
& -\left|\left\{(i, j) \in Q_{n-1} \mid F(i, j) \in \mathcal{F}_{4}\right\}\right| \\
\geq & 3\left|Q_{n-3}\right|-\left|Q_{n-1} \backslash Q_{n-3}\right| \\
\geq & 3\left|Q_{n}\right|-(16 n-24),
\end{aligned}
$$

and therefore

$$
\frac{\left|C \cap Q_{n}\right|}{\left|Q_{n}\right|} \geq \frac{3}{4}-\frac{4 n-6}{(2 n+1)^{2}},
$$

and the claim follows. 


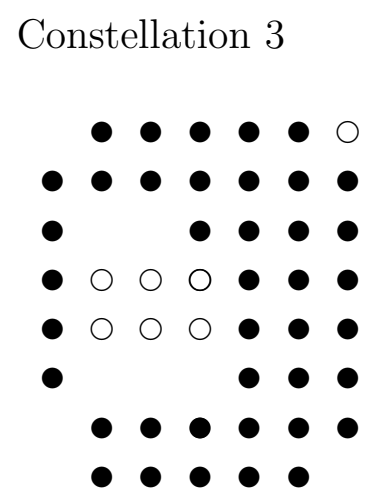

Constellation 4

Figure 3: Two more constellations.

\subsection{Codes with $t=2$ and $r>1$}

Now let us consider $r>1$.

Theorem 8 Assume that $r>1$. There is an $r$-identifying code that is robust against two unknown edge deletions in $K$ and has density $\frac{1}{4 r-4}$. The density of an r-identifying code in $K$ that is robust against two unknown edge deletions is at least $\frac{1}{4 r-2}$.

Proof. The lower bound follows from Theorem 11.

The code

$$
C=\{(x, y) \mid y-x \equiv 0 \quad(\bmod 2 r-2), x \equiv 0(\bmod 2)\}
$$

is as required, as can be seen using an argument similar to the one in the proof of Theorem 5 (recall that whenever Lemma 4 guarantees three disjoint paths to a codeword not all of them can be cut by two edge removals).

\subsection{The case $t \geq 3$}

There are no codes in $K$ that are $r$-identifying and robust against three or more unknown edge deletions. Take any code $C$, and let $p=(0,0)$ and $q=(1,0), K^{\prime}$ be the graph obtained from $K$ by deleting the three edges from $(0,0)$ to $(-1,-1),(-1,0)$ and $(-1,1)$, and $K^{\prime \prime}$ the graph obtained from $K$ by deleting the three edges from $(1,0)$ to $(2,-1)$, $(2,0)$ and $(2,1)$. Then

$$
B_{r}\left(K^{\prime} ; p\right)=B_{r}(K ; p) \backslash\{p+(-r,-r), p+(-r,-r+1), \ldots, p+(-r, r)\},
$$

and likewise

$$
B_{r}\left(K^{\prime \prime} ; q\right)=B_{r}(K ; q) \backslash\{q+(r,-r), q+(r,-r+1), \ldots, q+(r, r)\},
$$

and we see that $I_{r}\left(K^{\prime} ; p\right)=I_{r}\left(K^{\prime \prime} ; q\right)$. 


\section{Known edge deletions}

In this section we concentrate on the other class of robust identifying codes, namely, on the ones that are robust against known edge deletions.

\subsection{The case $t \geq 6$}

There are no codes in $K$ that are $r$-identifying and robust against six or more known edge deletions. Take any code $C$, and let $p=(0,0)$ and $q=(1,0)$, and let $K^{\prime}$ be the graph obtained from $K$ by deleting the three edges from $(0,0)$ to $(-1,-1),(-1,0)$ and $(-1,1)$, and the three edges from $(1,0)$ to $(2,-1),(2,0)$ and $(2,1)$. Then $I_{r}\left(K^{\prime} ; p\right)=I_{r}\left(K^{\prime} ; q\right)$.

\subsection{Codes with $r=1,1 \leq t \leq 5$}

The following theorem immediately follows from [21].

Theorem 9 The smallest possible density of a 1-identifying code in $K$ that is robust against $t$ known edge deletions is $\frac{t+1}{6}$ for $t=1,2,3,4,5$.

Proof. If $C$ is a 1-identifying code that is robust against $t$ known edge deletions, then clearly the symmetric difference $B_{1}((x, y)) \triangle B_{1}((x+1, y))$ (which consists of six points) must contain at least $t+1$ codewords, and the lower bound on the density follows. It is shown in [21, Theorem 2] that a code with density $\frac{t+1}{6}$ exists in $K$ that is 1 -identifying in all graphs obtained by deleting and/or adding at most $t$ edges to $K$.

\subsection{Codes with $t=1, r>1$}

Theorem 10 Assume that $r>1$. The smallest possible density of an $r$-identifying code in $K$ that is robust against one known edge deletion is $\frac{1}{4 r}$.

Proof. In $K$ the symmetric difference $S:=B_{r}((x, y)) \triangle B_{r}((x+1, y))$ consists of $2(2 r+1)$ vertices. We use Theorem 3 and assign the weight $1 / 2$ to the points $(x-r, y-r)$, $(x-r, y+r),(x+1+r, y+r)$ and $(x+1+r, y-r)$ (to which there is only one path of length $r$ from $(x, y)$ or $(x+1, y))$ and the weight 1 to the other $4 r-2$ points. If there are no codewords among the $4 r-2$ points, then there must be two codewords among the four points with weight $1 / 2$. Thus $m=1$ in Theorem 3 .

The upper bound immediately follows from Theorem 5. 


\subsection{Codes with $t=2, r>1$}

Theorem 11 Assume that $r>1$. There is an $r$-identifying code in $K$ that is robust against two known edge deletions and has density $\frac{1}{4 r-4}$. If $C$ is any $r$-identifying code in $K$ that is robust against two known edge deletions, then its density is at least $\frac{1}{4 r-2}$.

Proof. The lower bound follows from Theorem 3 as follows. Take $p=(x, y), q=$ $(x+1, y)$, and $S:=B_{r}((x, y)) \triangle B_{r}((x+1, y))$ in $K$, and assign the points $p+(-r,-r)$, $p+(-r,-r+1), p+(-r, r-1), p+(-r, r)$ as well as the points $q+(r,-r), q+(r,-r+1)$, $q+(r, r-1), q+(r, r)$ with weight $1 / 2$ and all the other points with weight 1 . The reasoning for $m=1$ goes analogously to the proof of the previous theorem.

The existence of such a code with density $1 /(4 r-4)$ follows from Theorem 8 .

\subsection{Codes with $t=3, r>1$}

Theorem 12 Assume that $r>1$. There is an $r$-identifying code in $K$ that is robust against three known edge deletions and has density $\frac{1}{2 r}$. If $C$ is any $r$-identifying code in $K$ that is robust against three known edge deletions, then its density is at least $\frac{1}{2 r+1}$.

Proof. Take $C=\left\{(x, y) \in \mathbb{Z}^{2} \mid y-x \equiv 0 \quad(\bmod 2 r)\right\}$. Assume that $p=\left(x_{p}, y_{p}\right)$ and $q=\left(x_{q}, y_{q}\right)$ are two different points in $\mathbb{Z}^{2}$. By symmetry, assume that $y_{p}>y_{q}$. If at most three edges have been deleted from $K$ to obtain $K^{\prime}$, we know that in $K^{\prime}$ at most one edge has been deleted from the half-plane $y \geq y_{p}$ or from the half-plane $y \leq y_{q}$. Without loss of generality, assume the former. If any of the $2 r-1$ points $p+(-r+1, r), p+(-r+2, r)$, $\ldots, p+(r-1, r)$ is in $C$, then by Lemma 4 this codeword is in $I_{r}\left(K^{\prime} ; p\right)$, but not in $I_{r}\left(K^{\prime} ; q\right)$; if not, then both $p+(-r, r)$ and $p+(r, r)$ are in $C$, and at least one of them belongs to $I_{r}\left(K^{\prime} ; p\right)$ but not $I_{r}\left(K^{\prime} ; q\right)$.

To see that $I_{r}\left(K^{\prime} ;\left(x_{0}, y_{0}\right)\right) \neq \emptyset$ for all $\left(x_{0}, y_{0}\right) \in \mathbb{Z}^{2}$ and all relevant graphs $K^{\prime}$, it suffices to notice that at most one edge with an end point in the half-plane $y>y_{0}$ has been deleted or at most one edge with an end point in the half-plane $y<y_{0}$ has been deleted, and use the same argument as above.

For the lower bound, let $(x, y) \in \mathbb{Z}^{2}$ be arbitrary. Consider the graph $K^{\prime}$ obtained by deleting the edges from $(x, y-1)$ to $(x-1, y-2),(x, y-2)$ and $(x+1, y-2)$. Then $B_{1}((x, y)) \triangle B_{1}((x, y-1))$ in $K^{\prime}$ consists of $2 r+1$ points, of which at least one has to be a codeword. The claim now follows from a standard density argument (i.e., in Theorem 3 we choose all the weights to be equal to one).

\subsection{Codes with $t=4, r>1$}

Theorem 13 Assume that $r>1$. There is an $r$-identifying code in $K$ that is robust against four known edge deletions and has density $\frac{1}{2 r-1}$. If $C$ is any $r$-identifying code in $K$ that is robust against four known edge deletions, then its density is at least $\frac{1}{2 r}$. 
Proof. The existence of such a code with density 1/(2r-1) follows from Theorem 14 .

For the lower bound, let $(i, j) \in \mathbb{Z}^{2}$ be arbitrary. Consider the graph $K^{\prime}$ obtained by deleting the edge from $(i, j)$ to $(i-1, j+1)$, and the edges from $(i, j-1)$ to $(i-1, j-2)$, $(i, j-2)$ and $(i+1, j-2)$. Then $B_{1}((i, j)) \triangle B_{1}((i, j-1))$ in $K^{\prime}$ consists of $2 r$ points, of which at least one has to be a codeword. The claim now follows as in the previous proof.

\subsection{Codes with $t=5$ and $r>1$}

In this case it is possible to find again the exact value of the optimal density.

Theorem 14 Assume that $r>1$. The smallest possible density of an $r$-identifying code in $K$ that is robust against five known edge deletions is $\frac{1}{2 r-1}$.

Proof. To obtain the lower bound, take $p=(x, y)$ and $q=(x+1, y)$, and let $K^{\prime}$ be the graph obtained by deleting the three edges from $p$ to $p+(-1,-1), p+(-1,0)$ and $p+(-1,1)$ and the two edges from $q$ to $q+(1,-1)$ and $q+(1,1)$. Then $I_{r}\left(K^{\prime} ; p\right) \subseteq I_{r}\left(K^{\prime} ; q\right)$ and $I_{r}\left(K^{\prime} ; q\right) \backslash I_{r}\left(K^{\prime} ; p\right)=\{q+(r,-r+1), q+(r,-r+2), \ldots, q+(r, r-1)\}$. Hence this $(2 r-1)$-element subset must contain at least one codeword and the lower bound on the density follows.

Take then

$$
C=\{(x, y) \mid y-x \equiv 0 \quad(\bmod 2 r-1)\} .
$$

Clearly, the density of $C$ equals $\frac{1}{2 r-1}$.

Assume that $p=\left(x_{p}, y_{p}\right)$ and $q=\left(x_{q}, y_{q}\right)$ are two different vertices; without loss of generality, $y_{p}>y_{q}$ (because the code is symmetric with respect to the line $y=-x$ ). Assume that $K^{\prime}$ is obtained from $K$ by deleting at most five edges. There are at most two edges missing from the half-plane $y \geq y_{p}$ or at most two edges missing from the half-plane $y \leq y_{q}$; say the former is true.

By Lemma 4, there are three edge-disjoint paths of length $r$ in $K$ from $p$ to each of the points $p+(-r+2, r), p+(-r+3, r), \ldots, p+(r-2, r)$, so no matter which edges have been deleted, these $2 r-3$ points belong to $B_{r}(p) \backslash B_{r}(q)$. There are two edge-disjoint paths of length $r$ from $p$ to $p+(r-1, r)$.

If $p+(r-1, r)$ does not belong to $B_{r}(p)$ in $K^{\prime}$, then one edge from each of these two paths has been deleted, and these are then the two deleted edges in the half-plane $y \geq y_{p}$. But then $p+(-r,-r)$ and $p+(-r+1, r)$ belong to $B_{r}(p)$ also in $K^{\prime}$, and we have found a subset $\{p+(-r, r), p+(-r+1, r), \ldots, p+(r-2, r)\}$ consisting of $2 r-1$ horizontally consecutive points, of which one is a codeword by the construction, and hence belongs to $I_{r}\left(K^{\prime} ; p\right) \backslash I_{r}\left(K^{\prime} ; q\right)$.

So assume that $p+(r-1, r)$ does belong to $B_{r}(p)$ in $K^{\prime}$. If also $p+(-r+1, r)$ belongs to $B_{r}(p)$ in $K^{\prime}$ we have again found a set of $2 r-1$ horizontally consecutive points that belong to $B_{r}(p) \backslash B_{r}(q)$, and are done. Assume therefore that $p+(-r+1, r)$ does not belong to $B_{r}(p)$ in $K^{\prime}$ There are two edge-disjoint paths from $p$ to $p+(-r+1, r)$, and 
one edge from each must have been deleted. But then $p+(r, r)$ belongs to $B_{r}(p)$, and we are again done.

In addition, $I_{r}\left(K^{\prime} ;\left(x_{0}, y_{0}\right)\right) \neq \emptyset$ for all $\left(x_{0}, y_{0}\right) \in \mathbb{Z}^{2}$ and all relevant graphs $K^{\prime}$. Indeed, notice that at most two edges with an end point in the half-plane $y>y_{0}$ has been deleted or at most two edges with an end point in the half-plane $y<y_{0}$ has been deleted, and use the same argument as above.

\section{References}

[1] Y. Ben-Haim, S. Litsyn, Exact minimum density of codes identifying vertices in the square grid, SIAM J. Discrete Math., 19 (2005), pp. 69-82.

[2] N. Bertrand, I. Charon, O. Hudry, A. Lobstein, Identifying and locating-dominating codes on chains and cycles, Europ. J. Combinatorics, 31 (2004), pp. 969-987.

[3] N. Bertrand, I. Charon, O. Hudry, A. Lobstein, 1-identifying codes in trees, Australas. J. Combin. 31 (2005), pp. 21-35.

[4] I. Charon, I. Honkala, O. Hudry, A. Lobstein, The minimum density of an identifying code in the king lattice, Discrete Math. 276 (2004), pp. 95-109.

[5] I. Charon, O. Hudry and A. Lobstein, Extremal cardinalities for identifying and locating-dominating codes in graphs, Discrete Math., 307 (2007), pp. 356-366.

[6] C. J. Colbourn, P. J. Slater and L. K. Stewart, Locating dominating sets in series parallel networks, Congr. Numer., 56 (1987), pp. 135-162.

[7] M. Daniel, S. Gravier and J. Moncel, Identifying codes in some subgraphs of the square lattice, Theoret. Comput. Sci., 319 (2004), pp. 411-421.

[8] A. S. Finbow and B. L. Hartnell, On locating dominating sets and well-covered graphs, Congr. Numer., 65 (1988), pp. 191-200.

[9] J. Gimbel, B. D. Van Gorden, M. Nicolescu, C. Umstead and N. Vaiana, Location with dominating sets, Congr. Numer., 151 (2001), pp. 129-144.

[10] S. Gravier, J. Moncel and A. Semri, Identifying codes of cycles, European J. Combin., 27 (2006), pp. 767-776.

[11] I. Honkala, An optimal robust identifying code in the triangular lattice, Ann. of Comb. 8 (2004), pp. 303-323.

[12] I. Honkala, A family of optimal identifying codes in $\mathbb{Z}^{2}$, J. Combin. Theory, Ser. A, 113 (2006), pp. 1760-1763.

[13] I. Honkala, On 2-edge-robust $r$-identifying codes in the king grid, Australas. J. Combin., 36 (2006), pp. 151-165.

[14] I. Honkala, M. Karpovsky, L. Levitin, On robust and dynamic identifying codes, IEEE Trans. Inform. Theory, 52 (2006), pp. 599-612.

[15] I. Honkala, T. Laihonen, On a new class of identifying codes in graphs, Information Processing Lett., 102 (2007), pp. 92-98. 
[16] I. Honkala, T. Laihonen, On identifying codes that are robust against edge changes, Inform. and Comput., 205 (2007), pp. 1078- 1095.

[17] I. Honkala, T. Laihonen, On vertex-robust identifying codes of level three, Ars Combin., to appear.

[18] M. G. Karpovsky, K. Chakrabarty, L. B. Levitin, On a new class of codes for identifying vertices in graphs, IEEE Trans. Inform. Theory 44 (1998), pp. 599-611.

[19] T. Laihonen, On optimal edge-robust and vertex-robust $(1, \leq l)$-identifying codes, SIAM J. Discrete Math, 18 (2005), pp. 825-834.

[20] T. Laihonen, On edge-robust $(1, \leq l)$-identifying codes in binary Hamming spaces, Int. J. Pure Appl. Math., 36 (2007), pp. 87-102.

[21] T. Laihonen, Optimal $t$-edge-robust $r$-identifying codes in the king lattice, Graphs Combin., 22 (2006), pp. 487-496.

[22] T. Laihonen, On robust identification in the square and king grids, Discrete Appl. Math., 154 (2006), pp. 2499-2510.

[23] A. Lobstein, http://perso.enst.fr/ lobstein/bibLOCDOMetID.html.

[24] J. Moncel, On graphs on $n$ vertices having an identifying code of cardinality $\left\lceil\log _{2}(n+1)\right\rceil$, Discrete Appl. Math., 154 (2006), pp. 2032- 2039.

[25] D. F. Rall and P. J. Slater, On location-domination numbers for certain classes of graphs, Congr. Numer., 45 (1984), pp. 97-106.

[26] S. Ray, R. Ungrangsi, F. De Pellegrini, A. Trachtenberg, D. Starobinski, Robust location detection in emergency sensor networks, Proceedings of INFOCOM 2003, San Francisco, March 2003.

[27] S. Ray, D. Statobinski, A. Trachtenberg and R. Ungrangsi, Robust location detection with sensor networks, IEEE Journal on Selected Areas in Communications, 22 (2004), pp. $1016-1025$.

[28] P. J. Slater, Domination and location in acyclic graphs, Networks, 17 (1987), pp. 55-64.

[29] P. J. Slater, Dominating and reference sets in a graph, J. Math. Phys. Sci., 22 (1988), pp. 445-455.

[30] P. J. Slater, Fault-tolerant locating-dominating sets, Discrete Math., 249 (2002), pp. 179-189. 